To cite this article:

Sarah Hean, Liz Anderson, Chris Green, Carol John, Richard Pitt \& Cath O'Halloran (2016) Reviews of theoretical frameworks: Challenges and judging the quality of theory application. Medical Teacher, 38:6, 613-620, DOI: 10.3109/0142159X.2015.1075648

To link to this article: $\underline{h t t p: / / d x . d o i . o r g / 10.3109 / 0142159 X .2015 .1075648 ~}$

\title{
Reviews of theoretical frameworks: challenges and judging the quality of theory application.
}

\section{Abstract}

\section{Background}

Rigorous reviews of available information, from a range of resources, is required to support medical and health educators in their decision making related to their educational practice.

$\underline{\text { Aim }}$

The aim of the paper is to highlight the importance of a review of theoretical frameworks specifically to supplement reviews that focus on a synthesis of the empirical evidence alone. Establishing a shared understanding of theory as a concept is highlighted as a challenge to these types of review and some practical strategies to achieving this are presented. The paper also introduces the concept of theoretical quality to the methodology of literature reviews, arguing that a critique of how theory is applied should complement the methodological appraisal of the literature in a review.

\section{Method}

We illustrate the challenge of establishing a shared meaning of theory through reference to experiences of an on-going review of this kind conducted in the field of interprofessional education (IPE) and use a high scoring paper selected in this review to illustrate how theoretical quality can be assessed. We focus on theories that apply to IPE curriculum design but the findings are transferable to all reviews of theoretical frameworks.

\section{Findings}


In reaching a shared understanding of theory as a concept, practical strategies that promote experiential and practical ways of knowing (e.g. small group work and piloting of all phases of the review protocol) are required in addition to more propositional ways of sharing knowledge. Concepts of parsimony, testability, operational adequacy and empirical adequacy are explored as concepts that establish theoretical quality.

\section{Conclusions}

Reviews of theoretical frameworks used in medical education are required to inform educational practice. Review teams should make time and effort to reach a shared understanding of the term theory. Theory reviews, and reviews more widely, should add an assessment of theory application to the protocol of their review method. 


\section{Introduction: the purpose of the BEME Review}

Medical educators, higher education institutions and professional bodies need to make informed decisions about their education practice and policy-making. The Best Evidence in Medical Education and Health Professions (BEME) Collaboration supports this decisionmaking through guiding the conduct of methodologically rigorous reviews that collect, appraise and synthesise the available information and evidence associated with a stated issue in health professional and medical education (http://www.bemecollaboration.org/).

We argue in this paper that BEME reviews should also support reviews of theoretical frameworks in addition to reviews that synthesise empirical evidence alone. In order to facilitate this process, we share in the paper some of the challenges of conducting a review of theoretical frameworks, based on the experiences of an on-going review of this kind being conducted in the field of interprofessional education (Hean et al., 2012). The paper also introduces the concept of theoretical quality to the protocol of a BEME review, arguing that this should complement the methodological appraisal of the literature when selecting the best evidence for medical education. Our intention is not to present a review of the theories currently in use in medical and health education but instead to begin a methodological debate on how available theory and its application can be best synthesised in a BEME review.

\section{Developing an intervention theory in medical and health education practice}

For stakeholders to make informed decisions in their educational practice, they need an understanding of the range of potential mechanisms through which educational interventions can lead to change in participants (e.g. attitudinal or behavioural changes). But these changes, intended and otherwise, are contingent on the context in which the intervention unfolds: a variety of conditions will trigger (or not) these mechanisms, in turn leading to a pattern of potential outcomes. The connection between mechanisms, context and outcomes together form the theory of the intervention (Pawson \& Tilley, 2004). Put another way, the theory of the intervention is a set of propositions that link concepts 
together through a rational argument. These statements predict, describe, explain, prescribe or organise a particular phenomenon (Fawcett \& Downs, 1992; Jary \& Jary, 1995; Walker \& Avant, 2005).

All educators will hold an intervention theory: their prediction as to why they believe an educational intervention should lead to change in students attitudes, knowledge or behaviours. These theories, whether implicitly or explicitly expressed, predict for whom the intervention will have an influence, how and why the intervention is expected to have this effect and in what conditions the intervention will lead to particular outcomes. Educators make these predictions/hypotheses/propositions informed by a range of information sources, including consultation with key stakeholders (e.g. students, teachers), previous educational models and experiences, professional body frameworks and information in the literature (Pawson and Tilley 2004).

BEME reviews focus on the synthesis of the information provided in the literature to help educators formulate their own intervention theory. To date BEME reviews focus on the empirical research and evaluation of the health and medical intervention or a component thereof. However, in developing the intervention theory, the mechanisms or logics behind an intervention (mechanisms), how these mechanisms are triggered or not by particular contextual conditions, the range of outcomes that may result and for whom, can also be informed by established theoretical frameworks that derive from the wider social science literature (broader education, sociology, psychology fields beyond the medical education sphere). Not all of these frameworks will have been tested in the medical education arena, and in the case of grand (as opposed in mid level or micro level theory- Merton, 1968) may have no empirical evidence to support them at all. They still provide a useful tool with which educators can make explicit why they believe an intervention might, under the right conditions, lead to certain outcomes. It is for the educator, assisted by evaluators and researchers, to test the viability of the programme theory put forward when the programme is initiated and in later iterations.

Having an intervention theory, as described above, is centrally important to the evidence base supporting any teaching and learning. It is an essential tool with which educators can 
define, predict, organise, explain and test their pedagogical approaches. Theory and practice are interdependent, with theory providing the structure and rigour to guide reflective educational practices and in turn, for practice to test out/validate and refine the theories they hold about what constitutes effective education. Some go so far as to state that practice, in this case educational practice, without a theory to guide it, amounts to malpractice (Eraut, 2003). We refer readers to Bordage (2009) for a more extended description of theory and conceptual frameworks can be used to clarify a problem and lead to practical solutions to problems in medical education in general.

\section{A theoretical review of interprofessional education as an illustration}

To illustrate the challenge of conducting these much needed theory reviews, we use a BEME review currently underway that explores the effective use of theory in the development of interprofessional education (IPE) (Hean et al., 2012). Many educators have written about the complexity of delivering IPE and the importance of strong theoretical underpinnings (Gilbert, 2005; Oandasan \& Reeves, 2005; Reeves et al. 2011; Thisthlethwaite, 2012; Barr, 2013; Reeves and Hean, 2013). Researchers in the field have mined other disciplines for theories that have utility in the field of IPE (Helme et al., 2005; Reeves et al., 2011; Hean et al., 2009) resulting in an abundance of varied theories drawn from a number of academic disciplines, including sociology, psychology, education and management. But the number and variety of theories presented to the IPE community in recent years has sometimes obscured, rather than clarified, the ways in which theory may contribute to the development of IPE curricula. This makes this field ripe for a theoretical type BEME review. A synthesis of the theories is now needed if the rigour of the field is to be advanced and if IPE educators are to be supported in making sense of the myriad of theories available.

A team of reviewers, members of the In-2-theory (interprofessional theory, scholarship and collaboration) network (Hean et al., 2013) responded to this need for a synthesis of theories in IPE and are in the process of conducting a BEME review (Hean et al., 2012) aiming to provide medical educators guidance on how to select and apply theories to IPE that are fit for purpose and enable educators to reflect on the why, rather than just the how, of designing, delivering and then evaluating the effectiveness of an interprofessional curriculum. 


\section{The meaning of theory: reaching a common understanding}

But in conducting this type of review, it became clear that one of the most central of challenges is the team reaching a common understanding of the meaning of the term theory. Reaching this common understanding is key to the inter rater reliability at all phases of the review protocol. These challenges will be familiar to any team of reviewers dealing with an intangible construct but strategies to overcome this are seldom articulated in the reporting of the review.

Theoretically, strategies to reach shared understanding rest on two concepts. Firstly, boundary crossing (Carlile, 2004) and secondly the different ways of knowing articulated by Heron and Reason (2008). Any review team will have members with a range of experiences and varied levels of theoretical competence. Some will be familiar with certain theories but not others and some will be more or less experienced in theory as a concept more generally (Hean et al., in press). Knowledge about any one individual theory and the "theory of theory" needs to transfer between members of the review team. Carlile (2004) describes the boundaries through which this knowledge must pass in this process, describing the need not only for the simple transfer of information between team members, but also the translation of knowledge into a common language and then the transformation of all knowledge, contributed by each team member, into an operationable definition of the term theory.

The strategies to transcend these boundaries, within the IPE theory review, is rooted in Heron and Reason's (2008) ways of knowing where four ways of acquiring knowledge are represented. The definition of theory written above is a propositional form of knowing, that is based on language and written description of the concept. The IPE review team members began to develop a common "knowing" of what theory meant by sharing the writing, for example, Fawcett \& Downs, (1992); Jary \& Jary, (1995); Walker \& Avant, (2005) and exploring Pawson and Tilley (2004)'s writing about programme theory. However, it was soon evident that this form of knowing was not enough to reach a common understanding of theory that could be reliably applied. We turned therefore to other ways of knowing for 
assistance: namely experiential and practical ways of knowing. Review members reached a consensus by doing tasks together (reminiscent of communities of practice described by Wenger (2002) (practical knowing); by holding regular workshops using exemplar pilot papers to work through together as a team during paper selection, quality assessment and extraction phases of the review. Experiential learning also took place when review members, more experienced in theory or a particular theory, worked together in pairs with less experienced members, articulating the review of any one particular paper, so that the less experienced member was able to learn experientially. Visualisation strategies were used at these times whereby the experienced reviewer highlighted what they had identified as theory in the paper, so less experienced partners could see how their partner was conceiving theory, rather than relying on their verbal description thereof (see Figure 1).

Another strategy to cement shared understanding of theory involved the translation of practical and experiential knowledge back into propositional knowledge. Hereby, members of the team were asked to write their reflections on what they felt the meaning of theory to be (propositional knowledge) drawing on their own previous knowledge of theory but also their understanding developed in their working experientially and practically with other members of the team in pairs and in workshops (see Figure 2 for an example of one reviewer's reflection).

Sharing knowledge through aesthetic, expressive methods such as art or dance, the final of Heron and Reasons (2008) ways of knowing (presentational knowing) is perhaps a tall order for a concept such as theory but should not be ruled out as means of reaching common understanding between participants.

It is critically important that time is set aside for the above processes to take place and for review leaders not to underestimate the importance or the time taken in achieving this shared meaning making. Regular workshops are required and regular actual and virtual contact is needed between reviewers between workshops and review meetings.

FIGURE 1 and 2 HERE

\section{Assessing the quality of application of theory}


Although the above discussion may have resonance with any review in which a key review concept is difficult to define, the task of assessing the quality, with which theory is applied, is unique to a theoretical review. In a conventional review of the evidence supporting a particular educational intervention, the assessment of methodological quality is key to the review team selecting papers of sufficient rigour so as to constitute good evidence in the field. A review of theory is distinct in this regard as the review is essentially exploring the effective use of theory in educational interventions. The focus is no longer on methodological quality but the quality with which theory has been used to underpin the curriculum or its evaluation. In other words, only those papers for which theories have been applied with the greatest quality will be included in the review. It is important to note here that it is not the quality of the theory itself that is in question but the quality of its application. This is the equivalent of stating that a survey/questionnaire, as a research method, is well established and valued but methodological quality is an issue if the application of these instruments is not suited to the research question or is applied in such a way that the data collected are not valid or reliable. Similarly, a particular theory may be relevant in explaining or predicting one element of an educational intervention but not another. It may be well established or acknowledged in some fields, sociology for example, but when applied to an educational intervention, it may be poorly or superficially described in reporting, the explicit link with the intervention may not be made obvious, and /or the theory may not be validated empirically within the specified educational context. We devote the rest of the paper to discussing these dimensions of theoretical quality in greater detail.

Whilst frameworks for assessing methodological quality are common place (e.g. BEME Collaboration, 2012; CASP, 2012) measures of theoretical quality are absent in systematic review methods. For this reason, the IPE review team developed their own framework to measure theoretical quality. The dimensions of this framework originate from those developed by Fawcett and Downes when assessing the links between theory and research (Fawcett \& Downs, 1992; Fawcett, 2005), namely the dimensions of parsimony, pragmatic adequacy, testability, operational and empirical adequacy. The meaning of each of these are discussed below. A tool, which may be used to assess these dimensions of quality theory 
application in a review protocol, is presented in Table 1. A worked example of the assessment of a paper that scored highly on all dimension of theory application quality is provided (Weaver et al., 2011).

\section{Pragmatic Adequacy (Is the utility of the theory for practice made clear?)}

Pragmatic adequacy means that the theory has clear utility for practitioners: when reading the report of the curriculum or its evaluation, practitioners should be able to see clearly how the theory can inform their working lives. By practice we mean the theory must have been used to underpin an interprofessional curriculum, the way it is delivered and/or the approach taken to its evaluation (Coles and Grant, 1985). Papers need to explicitly address the questions of who is the theory useful for and how can they use this theory to inform their working practice. In Table 1 for example, complexity theory underpins the analysis of focus groups exploring the experiences of a committee tasked with developing an IPE programme. The way in which Weaver et al (2011) have applied complexity theory in this study shows this theory to have clear relevance for both researchers and curriculum developers. For researchers, the theory has been used to underpin an analytical framework to analyse the transcripts of focus groups. For curriculum developers, the principles and conditions of complexity theory are clearly linked to ways in which these committees can be effectively run in the future.

Pragmatic adequacy is a key selection criterion for papers. There are many highly interesting and academically sophisticated papers exploring and developing theory but if the discussion remains at an abstract level with no clearly articulated link to education or research practice, then the paper should not be reviewed further. This is because the objective of a theory review is to provide medical and health educators with guidance on how best to use theories to guide their practice. A BEME theory review will synthesise the range of theories, where and how these are applied and tested in the sample of those papers in which theory has been applied with sufficient quality. 


\section{Parsimony (Is the theory concisely and clearly described?)}

Parsimony means that theories are clearly but concisely described in reporting. In many reports on IPE curricula, for example, the theory underpinning the curriculum is not described. It is then uncertain if the curriculum has no theoretical foundation, or whether this theory was simply not made explicit. Alternatively, theory may be referred to in the report but only nominally in passing with no explanation of what the theory entails or how it has been applied. Theories used in practice should be expressed clearly and concisely in reports, so as to engage readers without alienating them with excess and confusing detail. Authors on the other hand must guard against an over simplification of the phenomenon being addressed (Fawcett \& Downs, 1992; Fawcett, 2005). Papers are assessed on the degree to which this balance is achieved. In Table 1, Weaver et al (2011) have achieved this balance by highlighting in accessible language the three main principles of complex systems and the five conditions necessary for learning within these systems. Parsimony is an important skill, especially when reporting in journals with limited word counts, and many authors fall into the trap of overlooking their theoretical frameworks in their writing altogether as they do not have space and skill to fully describe the theoretical underpinnings of their curriculum or evaluation sufficiently and succinctly.

\section{Testability (Are clear propositions, derived from the theory, clearly presented?)}

Key concepts that form the components of the theory and the proposed relationships between them should be clearly articulated. So for example in Table 1, the three main principles of complexity theory are spelt out (i.e. that in these systems, knowledge is emergent, that the system is self-organizing, more than the sum of its parts and nested) in addition to the five conditions required for collective learning to take place within this complex system (e.g. decentralsied control, internal diversity). Weaver et al (2011) clearly propose that the IPE Committee is a complex system, that as such can be viewed from the perspective of the three principles of a complex system, collective learning and the conditions required for this.

Operational Adequacy (Is there a clear link between the propositions and the research method used to test or use the theory?) 
This criterion is fulfilled if the proposition/hypothesis created from the theory is then tested or applied with an appropriate research design and method. Weaver et al (2011) (Table 1) for example, clearly demonstrate how the dimensions of complexity theory and learning conditions have developed an analytical framework for a content analysis of the transcripts. Focus groups as a method are themselves a reasonable method with which to explore the application of complexity theory to the IPE committee being investigated.

\section{Empirical Adequacy}

This means that the empirical data collected is congruent with the theory that underpins the study. In more inductive studies, a clear theory must be articulated that explains a component of IPE and this theory must fit well with the themes arising from the analysis of interviews/focus groups conducted with study participants. In Table 1, for example, Weaver et al. (2011) are able to demonstrate how quotes within the focus groups within the IPE committee fitted with the dimensions of complexity theory. As researchers, they use this match to articulate and discuss the experiences of the IPE committee, providing an improved understanding and explanation of the original focus group data collected from committee participants. With their practitioner hat on, they use the theory informed analysis to compile a set of theory and evidence informed guidelines with which effective committees may be run in the future.

In reality, the above dimensions of theoretical quality described here are strongly interdependent, i.e. papers that have the most pragmatic adequacy are those that are clearly and simply articulated (parsimony), lay out clear propositions (testability), use appropriate methods to derive or test these propositions (operational adequacy) then report data that is congruent with the theoretical approach being utilised (empirical adequacy). This interdependence is reflected practically in the questions in the quality tool (Table 1) where single questions often capture multiple elements of theoretical quality.

\section{Conclusion}


In this paper we have argued for the rigorous review of theoretical frameworks used in the health and medical education literature. These reviews would be an important resource to support medical and health educators in their decision making when drawing on established theoretical frameworks that, in combination with their own practice experiences, create their context-specific intervention theory.

We discuss how review teams conducting a theory review should pay particular attention to developing a shared understanding of theory as a concept early on in the review process and we offer some practical strategies that promote experiential and practical ways of knowing (e.g. small group work and piloting of all phases of the review protocol) that go beyond agreeing a written definition of theory that relies on propositional ways of sharing knowledge.

Finally, we suggest that assessing the quality of theory application is central to the inclusion/exclusion of papers in a theory review and present the concepts of pragmatic adequacy, parsimony, testability, operational adequacy and empirical adequacy as dimensions of quality.

We recommend that the theory quality assessment tool (Table 1) be applied to future theory reviews. It should also be included as an extra quality dimension in the protocols of rigorous literature reviews more widely. We also recommend that the assessment tool not only be used as part of a review protocol but that it be used as a tool in the training of health and medical educators to help them develop their theoretical competence and their ability to develop, apply and evaluate their intervention theory explicitly in their educational practice.

The theoretical quality tool is a work in progress, however. It needs further refinement through validation in other educational fields other than IPE. BEME reviews are practitioner-focussed endeavours, and hence the focus on pragmatic value as a key inclusion criterion. We acknowledge this means the loss of many papers that still have high academic value and that make a contribution to theoretical development in the field. Pragmatic value is the only exclusion criterion and tool deliberately does not excluded nonempirical papers. Although these papers will score low on operational and empirical adequacy, an articulation of the intervention theory and how this applies to education 
practice, still has pragmatic value for the reader. So, not all levels of quality need to be achieved but whilst this encourages educators to articulate their intervention theory explicitly, there should be with the proviso that these intervention theories need to be tested in the future.

We have focused heavily in this paper on the deductive application of established theoretical frameworks to the area of literature being reviewed. We have not dwelt on the generation of theory inductively, either empirically through grounded theory approaches or in non empirical work where authors may explore the mechanisms at play in their own curriculum models, models based on their own personal, practice based experiences. Future tools are required that assess the quality of theory production in these contexts.

This paper has not presented the theories that are best suited for health and medical education. The list is potentially endless. We are more interested in the manner with which theory is applied and reported generically. We are recommending the exclusion of some papers from a review and not the exclusion of some theories from education practice. Excluding theories from discussion that have not been applied with sufficient quality does not mean that these theories are not worth pursing but that the method, with which these have been applied to inform practice, requires development. It may follow, that when a range of theory reviews are complete, that there may be certain theories that do not achieve empirical adequacy under a certain set of condition and that the theory itself will be put aside. However, we need to keep at the back of our mind that these theories will be proven or disproven under a particular set of conditions only and that these may have validity in other contexts. But, at this stage of our understanding of theory, at least in the IPE context, the problem faced currently is not that we have particular theories that are more or less relevant, but that most papers accept uncritically the truth of the theory they apply and that too few papers are reporting the empirical testing of theories in the first place. 


\section{PRACTICE POINTS}

1. When conducting a BEME review, spend time ensuring that all members of the team have a common understanding of key terms within the review. Add experiential and practical exercises to facilitate common understanding of the term theory rather than relying on a written or propositional definitions alone.

2. When conducting a BEME review that includes a review of theoretical frameworks add an extra phase to the protocol assessing the quality with which theory is applied.

3. When designing, delivering, evaluating or reporting educational interventions:

- articulate the theoretical framework explicitly, clearly but concisely

- lay out clear propositions for practice derived from the theory

- Use appropriate methods to test these propositions in practice, adjusting practice in light of these findings.

REFERENCES (to follow)

Barr H. 2013. Toward a theoretical framework for interprofessional education. J Interprofess Care 27(1):4-9[Taylor \& Francis Online], [Web of Science ${ }^{\circledR}$ ]

BEME Collaboration. 2012. BEME Coding Sheet - BEME Collaboration. [Accessed 01 July 2015] Available from www.bemecollaboration.org/downloads/749/beme4_appx1.pdf

Bordage G. 2009. Conceptual frameworks to illuminate and magnify. Med Educ 43(4):312319[CrossRef], [PubMed], [Web of Science ${ }^{\circledR}$ ]

Carlile PR. 2004. Transferring, translating, and transforming: An integrative framework for managing knowledge across boundaries. Organ Sci 15(5):555-568[CrossRef], [Web of Science $\left.{ }^{\circledR}\right]$

CASP. 2012. Critical Appraisal Skills Programme. [Accessed July 2015] Available from http://www.casp-uk.net/find-appraise-act/appraising-the-evidence 
Coles C, Grant GJ. 1985. Curriculum evaluation in medical and health-care education. Med Educ 19:405-422[CrossRef], [PubMed], [Web of Science ${ }^{\circledR}$ ]

Eraut M. 2003. The many meanings of theory and practice. Learn Health Soc Care 2(2):6165[CrossRef]

Fawcett J. 2005. Criteria for evaluation of theory. Nurs Sci Q 18(2):131-135[CrossRef], [PubMed], [Web of Science ${ }^{\circledR}$ ]

Fawcett J, Downs FS. 1992. The relationship of theory and research. 2nd ed. Philadelphia, PA: F.A. Davis

Hean S, Anderson E, Bainbridge L, Clark PG, Craddock D, Doucet S, Oandasan I. 2013. IN-2THEORY - Interprofessional theory, scholarship and collaboration: A community of practice. J Interprofess Care 27(1):88-90[Taylor \& Francis Online], [Web of Science ${ }^{\circledR}$ ]

Hean S, Craddock D, Hammick M. 2012a. Theoretical insights into interprofessional education: AMEE Guide No. 62. Med Teach 34(2):e78-101[Taylor \& Francis Online], [Web of Science $\left.{ }^{\circledR}\right]$

Hean S, O'Halloran C, Craddock D, Pitt R, Anderson L, Morris D. 2012b. A systematic review of the contribution of theory to the development of effective interprofessional curricula in medical education: Initial Review Protocol. Dundee, Scotland: Proposal to Best Evidence Medical Education (BEME) Collaboration. [Accessed 1 July 2015] Available from www.bemecollaboration.org

Hean S, Doucet S, Ball V, Anderson L, Bainbridge L, Baldwin C, Green C, Pitt R, Snyman S, Schmidtt $\mathrm{M}$, et al. in press. Moving from atheoretical to theoretical approaches to interprofessional client-centred collaborative practice. In: Orchard C, Herbert C, Bainbridge L, editors. Interprofessional client-centred collaborative practice - What does it look like? How can it be achieved? New York: Nova Helme M, Jones I, Colyer H. 2005. The theory-practice relationship in interprofessional education. London: HSAP Subject Centre 
Heron P, Reason J. 2008. Extending epistemology within a cooperative inquiry. In: Reason P, Bradbury $\mathrm{H}$, editors. The SAGE handbook of action research: Participative inquiry and practice. London: Sage[CrossRef]

Jary DJ, Jary J. 1995. Collins dictionary of sociology. Glasgow: Collins

Merton RK. 1968. Social theory and social structure. New York: Simon and Schuster

Pawson R, Tilley N. 1997. Realistic evaluation. London: Sage

Reeves S, Hean S. 2013. Why we need theory to help us better understand the nature of interprofessional education, practice and care. J Interprofess Care 27(1):1-3[Taylor \& Francis Online], [Web of Science ${ }^{\circledR}$ ]

Thistlethwaite J. 2012. Interprofessional education: A review of context, learning and the research agenda. Med Educ 46(1):58-70[CrossRef], [PubMed], [Web of Science ${ }^{\circledR}$ ]

Walker L, Avant KC. 2005. Strategies for theory construction in nursing. 4th edition. Upper Saddle River, NJ: Pearson Prentice Hall

Weaver L, Mcmurtry A, Conklin J, Brajtman S. 2011. Harnessing complexity science for interprofessional education development: A case study. J Res Interprofess Pract Educ 2(1):100-121

Wenger E. 2002. Cultivating cultivating communities communities of of practice practice a quick start-up guide guide. [Accessed 01 July 2015] Available from http://ec.europa.eu/employment_social/equal_consolidated/data/document/0709-copguide_en.pdf 